\title{
ON MAJORIZATION AND NORMALITY OF OPERATORS
}

\author{
MEHDI RADJABALIPOUR
}

\begin{abstract}
Recent results of C. R. Putnam are used to find some conditions for normality of operators. The emphasis is on the classes of spectral operators (defined by N. Dunford) and $M$-hyponormal operators (defined by J. G. Stampfli).
\end{abstract}

Throughout the paper by an operator we mean a bounded linear transformation defined on a fixed Hilbert space $H$.

We will study pairs $(T ; D)$ of operators satisfying the following Putnam condition:

$$
\left\{\begin{array}{l}
D \geqslant 0, \\
(T-z)(T-z)^{*} \geqslant D^{2} \text { for all } z \in \mathbf{C} .
\end{array}\right.
$$

Putnam [6], [7] shows that if a pair $(T ; D)$ of operators satisfies condition (1) and if $x$ is a vector in the range of $D$, then there exists a bounded function $w: \mathbf{C} \rightarrow H$ such that $(T-z) w(z) \equiv x$. We give a short proof of this result and show that if $T$ is an $M$-hyponormal operator, then $\left(T^{*} ; k\left|T^{*} T-T T^{*}\right|\right)$ satisfies (1) for some $k>0$. This fact is then used to improve some of the results of B. L. Wadhwa [11] on $M$-hyponormal operators. For instance, we prove that if $T$ is an $M$-hyponormal operator, if $S$ is a subspectral or a dominant operator, if $W$ is a quasi-affinity, and if $W T^{*}=S W$, then $T$ is normal. (See below for the definitions.) It also follows from some results of Putnam [7] that $M$-hyponormal, compact operators and certain other $M$ hyponormal operators are necessarily normal. The paper is concluded by discussing some general properties of dominant operators.

Main results. We first give a new proof of the following result due to Putnam [6, Theorem 1].

Theorem 1. Let $(T ; D)$ satisfy condition (1) and let $x=$ Dy for some $y \in H$. Then there exists a function $w(z): \mathbf{C} \rightarrow H$ such that $(T-z) w(z) \equiv x$ and

$$
\|w(z)\|^{2} \leqslant \int_{0+}^{\infty} u^{-2} d\left\|E_{u} x\right\|^{2}<\infty \quad(z \in \mathbf{C}),
$$

Received by the editors February 18, 1976 and, in revised form, May 5, 1976.

AMS (MOS) subject classifications (1970). Primary 47B20, 47B40, $47 \mathrm{~B} 47$.

Key words and phrases. Normal operator, spectral operator, $M$-hyponormal operator, quasisimilarity. 
where $E$ is the resolution of the identity for $D$.

Proof. By [3, Theorem 1], for each $z \in \mathbf{C}$ there exists a contraction operator $C_{z}$ such that $D=(T-z) C_{z}$. Let $w(z)=C_{z} y$. It follows that $(T-z) w(z) \equiv x$ and that

$$
\|w(z)\|^{2} \leqslant\|y\|^{2}=\int_{0+}^{\infty} u^{-2} d\left\|E_{u} x\right\|^{2}<\infty \quad(z \in \mathbf{C}) .
$$

The definitions and some properties of dominant operators and $M$-hyponormal operators are given in [9] and [11]. An operator $T$ is called a dominant operator if $(T-z) H \subseteq(T-z)^{*} H$ for all $z \in \mathbf{C}$, or, equivalently, if for each $z \in \mathrm{C}$ there exists a positive number $M_{z}$ such that

$$
(T-z)(T-z)^{*} \leqslant M_{z}(T-z)^{*}(T-z)
$$

(see [3]). If, in (2), the constants $M_{z}$ are bounded by a positive number $M$, then $T$ is called an $M$-hyponormal operator. Note that $T$ is hyponormal if and only if it is 1-hyponormal.

In the following, by a subspectral operator we mean the restriction of a spectral operator to an invariant subspace.

COROllary 1. Let $(T ; D)$ satisfy condition (1). Let $W$ be an operator with a finite-dimensional null space. Assume $W T=S W$ for some dominant or subspectral operator $S$. Then $D=0$.

The corollary is essentially proved in [4], [8] and [10]; actually, if we assume with no loss of generality that $H$ is separable, then the proof is an imitation of that of Theorem 3 of [8].

Theorem 2. Let $A$ and $B$ be two operators and let $M$ be a positive number. Assume for $|z| \leqslant\|B\|+1$ there exists a bounded family $S_{z}$ of operators such that $S_{z} B=B S_{z}$ and

$$
\left(A-S_{z}\right)\left(A-S_{z}\right)^{*} \leqslant M^{2}(B-z)(B-z)^{*} .
$$

Then $\left(B ; k\left|A^{*} B^{*}-B^{*} A^{*}\right|\right)$ satisfies condition (1) for some $k>0$. In particular, if $T$ is an $M$-hyponormal operator, then $\left(T^{*} ; k\left|T^{*} T-T T^{*}\right|\right)$ satisfies (1) for some $k>0$.

Proof. By [3] there exists a family $C_{z}(|z| \leqslant\|B\|+1)$ of operators such that $\left\|C_{z}\right\| \leqslant M$ and $A-S_{z}=(B-z) C_{z}$. Thus, for $|z| \leqslant\|B\|+1$,

$$
\begin{aligned}
A B-B A & =\left(A-S_{z}\right)(B-z)-(B-z)\left(A-S_{z}\right) \\
& =(B-z)\left[C_{z}(B-z)-\left(A-S_{z}\right)\right] ;
\end{aligned}
$$

and, for $|z|>\|B\|+1$,

$$
A B-B A=(B-z)(B-z)^{-1}(A B-B A) .
$$


Hence $k^{2}\left|A^{*} B^{*}-B^{*} A^{*}\right|^{2} \leqslant(B-z)(B-z)^{*}$ for all $z \in \mathrm{C}$, where $1 / k$ is the larger of

$$
\sup _{|z| \leqslant\|B\|+1}\left\|C_{z}(B-z)-\left(A-S_{z}\right)\right\| \text { and } \sup _{|z| \geqslant\|B\|+1}\left\|(B-z)^{-1}(A B-B A)\right\|
$$

(again use [3]). The rest of the proof is obvious.

The following corollary of Theorem 2 generalizes Theorem 1 of [10] and Theorem 6 of [11].

COROLlARY 2. Let $T$ be an M-hyponormal operator and let $W$ be an operator with a finite-dimensional null space. Assume $W T^{*}=S W$ for some dominant or subspectral operator $S$. Then $T$ is normal.

The proof follows from Corollary 1 and Theorem 2.

COROllaRY 3. If an $M$-hyponormal operator $T$ is similar to a spectral operator, then $T$ is normal.

In view of [7, Theorem 2] the following is an immediate corollary of our Theorem 2; it improves Theorem 5 of [11]. For the notions and properties of analytic and continuous capacities, see [7] and the references cited here. Note that a compact countable set has analytic capacity zero and, by Morera's theorem, a rectifiable curve has continuous capacity zero.

Corollary 4. Let $T$ be an M-hyponormal operator. Assume that the continuous capacity of $\sigma(T)$ and the analytic capacity of the closure of $\sigma_{p}\left(T^{*}\right)$ are zero. Then $T$ is normal.

The following corollary simplifies the construction of a dominant operator that is not $M$-hyponormal for any $M>0$, given in [9, Example 2].

COROllary 5. A quasinilpotent, M-hyponormal operator is zero. Therefore if $\left\{e_{n}\right\}_{-\infty<n<\infty}$ is an orthonormal basis for $H$ and if $T$ is the bilateral weighted shift defined by $T e_{n}=2^{-|n|} e_{n+1}$, then both $T$ and $T^{*}$ are compact, quasinilpotent and dominant, but are not $M$-hyponormal for any $\boldsymbol{M}>0$.

Note. In view of Corollary 2 , if an operator $T$ and its adjoint are both $M$ hyponormal, then $T$ is normal.

COROLlaRY 6. A compact, M-hyponormal operator is normal.

An operator $A$ is said to be a quasi-affine transform of an operator $B$, denoted by $A \prec B$, if there exists an injective operator $W$ with dense range such that $W A=B W ; A$ and $B$ are said to be quasi-similar if $A \prec B \prec A$. The following theorem gathers some of the conclusions of the relation $T^{*} \prec S$, when $T$ and $S$ are normal, spectral, hyponormal, etc.; some parts of the theorem have been known before and proper references are given in the proof. 
THEOREM 3. Let $T$ and $S$ be two operators such that $T^{*} \prec S$. The following statements are true.

(a) If $T$ is $M$-hyponormal and $S$ is dominant then $T^{*}$ and $S$ are unitarily equivalent normal operators.

(b) If $T$ is $M$-hyponormal and $S$ is subspectral then $T$ is normal and $S$ is similar to $T^{*}$.

(c) If $T$ and Sare subspectral then both $T^{*}$ and $S$ are spectral but not necessarily similar to each other.

(d) Statements (a) and (b) are false if $T$ is assumed to be only a dominant operator.

Proof. (a) The normality of $T$ follows from Corollary 2; the normality of $S$ and its equivalence with $T$ follows from [9, Theorem 1].

(b) Again here the normality of $T^{*}$ follows from Corollary 2 and the rest follows from [4, Theorem 3].

(c) The fact that $T$ and $S$ are spectral follows from [4, Theorem 3]; in [1] a nilpotent operator is constructed which is, of course, quasi-similar to its adjoint but not similar to it.

(d) Let $T$ be the bilateral weighted shift of Corollary 5 and let $S=T^{*}$. Obviously $T^{*} \prec S$ and both $T$ and $S$ are dominant and quasinilpotent.

REMARK 1. In view of part (d) of the theorem, statements (a), (b) and (c) seem to be the most that can be said along these lines; for special cases see [4], [8], [10] and [11].

REMARK 2 . Note that if $A$ is any cyclic operator of norm less than 1 and if $S$ is the unilateral shift, then $S \prec A$ [2, Theorem 2.1]; therefore Theorem 3 does not remain true if the relation $T^{*} \prec S$ is replaced by the relation $S \prec T^{*}$ (see also [9, Example 1]).

It is known that, as in the case of hyponormal operators, every eigenvalue of a. dominant operator is reducing; the following theorem shows that dominant (and $M$-hyponormal) operators share a stronger form of this property with their hyponormal brethren.

THEOREM 4. A normal part of a dominant operator is reducing.

Proof. Let $T$ be a dominant operator and let $N$ be a normal part of $T$. Let $\Re$ be the domain of $N$ and write

$$
T=\left[\begin{array}{cc}
N & A \\
0 & B
\end{array}\right]_{\mathscr{R}^{\perp}}^{\Re}
$$

Let $y$ be an arbitrary vector in $\Re^{\perp}$. Since $(T-z) H \subseteq(T-z)^{*} H(z \in \mathrm{C})$, $A y=(N-z)^{*} u_{z}$ for some $u_{z} \in \Re$. Choose $v_{z}$ such that $(N-z)^{*} u_{z}=$ $(N-z) v_{z}$. It follows that $A y=(N-z) v_{z}$ and thus $A y \in \bigcap_{z \in \mathbf{C}}(N-z) \Re$. Hence by [5, Theorem 1], $A y=0$ and therefore $A=0$.

Note. Theorem 4 is well known for hyponormal operators. 
REMARK 3. Let $T$ and $D$ be a pair of operators satisfying the following conditions:

(3) $D \geqslant 0$,

(4) $D H \subseteq(T-z) H$ for all $z \in \mathbf{C}$.

In view of [3], condition (4) is equivalent with

$\left(4^{\prime}\right) D^{2} \leqslant M_{z}(T-z)\left(T^{*}-\bar{z}\right)$ for all $z \in \mathbf{C}$,

where $M_{z}$ is a positive number for each $z$; hence condition (1) implies conditions (3) and (4). If $T$ is a subnormal operator and $(T ; D)$ satisfies $(3)$ and (4), then $D=0$ [5]. Moreover if $T$ is a dominant operator, then $\left(T^{*}\right.$, $\left|T^{*} T-T T^{*}\right|$ ) satisfies (3) and (4) (the proof is similar to that of Theorem 2).

REMARK 4. For an operator $T$ and a closed subset $\delta$ of $\mathbf{C}$ we define the (analytic) spectral manifold $X_{T}(\delta)$, the bounded spectral manifold $Y_{T}(\delta)$, and the unconditional spectral manifold $Z_{T}(\delta)$ as follows:

$X_{T}(\delta)=\left\{x \in H:(T-z) f_{x}(z) \equiv x\right.$ for some analytic

$$
\text { function } \left.f_{x}: \mathbf{C} \backslash \delta \rightarrow H\right\},
$$

$Y_{T}(\delta)=\left\{x \in H:(T-z) f_{x}(z) \equiv x\right.$ for some function

$f_{x}: \mathbf{C} \backslash \delta \rightarrow H$ that is bounded on compact subsets of $\left.\mathbf{C} \backslash \delta\right\}$,

$Z_{T}(\delta)=\bigcap_{z \notin \delta}(T-z) H$.

It is clear that $X_{T}(\delta) \subseteq Y_{T}(\delta) \subseteq Z_{T}(\delta)$. If a pair $(T ; D)$ satisfies (3) and (4), then $D H \subseteq Z_{T}(\varnothing)$; if $(T ; D)$ satisfies (1) then $D H \subseteq Y_{T}(\varnothing)$. In the following we raise some questions concerning the relations between the various spectral manifolds of dominant or $M$-hyponormal operators.

(a) It is known [8] that if $T$ is hyponormal then $X_{T}(\delta)$ is closed for all closed sets $\delta$. Is a similar result true for an $M$-hyponormal or a dominant operator?

(b) In view of [4, Corollary 1] and [10] if $T$ is a subspectral or a dominant operator, then $X_{T}(\delta)=Y_{T}(\delta)$ for all closed sets $\delta$; it follows from [5] that for a subnormal operator $T, X_{T}(\delta)=Z_{T}(\delta)$ for all closed sets $\delta$. The dominant, quasinilpotent operator $T$ of Corollary 5 has the property that $X_{T}(\varnothing)$ $=Y_{T}(\varnothing)=\{0\}$ but $Z_{T}(\varnothing)=T H$. Is $Z_{T}(\delta)=X_{T}(\delta)$ for $M$-hyponormal operators $T$ ? The question is unsettled even for hyponormal operators. Some results of [8] and specially of [10] support the conjecture that the question may have an affirmative answer.

REMARK. C. K. Fong has independently shown that a spectral, $M$-hyponormal operator has an orthogonal resolution of the identity; this result is improved by our Corollary 3.

\section{REFERENCES}

1. C. Apostol, R. G. Douglas and C. Foias, Quasi-similar models for nilpotent operators (to appear).

2. C. A. Berger and B. I. Shaw, Hyponormality: its analytic consequences (to appear).

3. R. G. Douglas, On majorization, factorization and range inclusion of operators on Hilbert 
space, Proc. Amer. Math. Soc. 17 (1966), 413-415. MR 34 \#3315.

4. C. K. Fong and M. Radjabalipour, On quasiaffine transforms of spectral operators, Michigan Math. J. (to appear).

5. C. R. Putnam, Ranges of normal and subnormal operators, Michigan Math. J. 18 (1971), 33-36. MR 43 \#2550.

6. Hyponormal contractions and strong power convergence, Pacific J. Math. 57 (1975), 531-538.

7.

8. M. Radjabalipour, Ranges of hyponormal operators, Illinois J. Math. (to appear).

9. J. G. Stampfli and B. L. Wadhwa, An asymmetric Putnam-Fuglede theorem for dominant operators, Indiana Univ. Math. J. (to appear).

10. - On dominant operators (to appear).

11. B. L. Wadhwa, M-hyponormal operators, Duke Math. J. 41 (1974), 655-660. MR 49 \#11302.

\section{Department of Mathematics, Dalmousie University, Halifax, Nova Scotia, Canada}

Current address: Department of Mathematics, Reza Shah Kabir University, P.O. Box 41-1811, Tehran, Iran 